\section{Heart Rate Variability Classification using Support Vector Machine and Genetic Algorithm}

\author{
Ashtiyani M. ${ }^{1}$, Navaei Lavasani S. ${ }^{1}$ Asgharzadeh Alvar A. ${ }^{1}$, \\ Deevband M. R. ${ }^{1 *}$
}

\begin{abstract}
Background: Electrocardiogram (ECG) is defined as an electrical signal, which represents cardiac activity. Heart rate variability (HRV) as the variation of interval between two consecutive heartbeats represents the balance between the sympathetic and parasympathetic branches of the autonomic nervous system.

Objective: In this study, we aimed to evaluate the efficiency of discrete wavelet transform (DWT) based features extracted from HRV which were further selected by genetic algorithm (GA), and were deployed by support vector machine to HRV classification.

Materials and Methods: In this paper, 53 ECGs including 3 different beat types (ventricular fibrillation (VF), atrial fibrillation (AF) and also normal sinus rhythm (NSR)), were selected from the MIT/BIH arrhythmia database. The approach contains 4 stages including HRV signal extraction from each ECG signal, feature extraction using DWT (entropy, mean, variance, kurtosis and spectral component $\beta$ ), best features selection by GA and classification of normal and abnormal ECGs using the selected features by support vector machine (SVM).
\end{abstract}

Results: The performance of the classification procedure employing the combination of selected features were evaluated using several measures including accuracy, sensitivity, specificity and precision which resulted in $97.14 \%, 97.54 \%, 96.9 \%$ and $97.64 \%$, respectively.

Conclusion: A comparative analysis with the related existing methods illustrates the proposed method has a higher potential in the classification of AF and VF. The attempt to classify the ECG signal has been successfully achieved. The proposed method has shown a promising sensitivity of $97.54 \%$ which indicates that this technique is an excellent model for computer-aided diagnosis of cardiac arrhythmias.

\section{Keywords}

Heart Rate Variability (HRV), Wavelet Transform, Genetic Algorithm (GA), Support Vector Machine (SVM)

\section{Introduction}

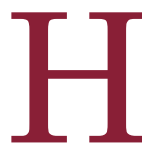
eart is a vital muscular organ which pumps oxygenated blood throughout vessels by recurring contractions. Any trouble in heart rhythm can be very hazardous. Although cardiac arrhythmia is one of the causes of death, it can be remedied if diagnosed on time $[1,2]$. Heart has four chambers including two lower chambers and two upper chambers. The two lower chambers are the ventricles (the right and the left ventricle) and the two upper chambers are called atria (the
${ }^{1}$ Department of Biomedical Engineering and Medical Physics, Faculty of Medicine, Shahid Beheshti University of Medical Sciences, Tehran, Iran

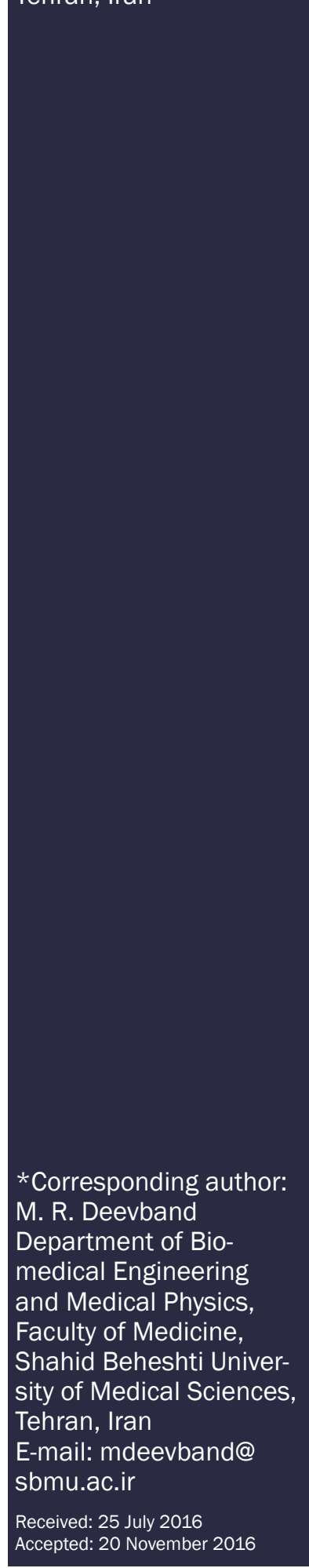


left and the right atrium). When the atria (two upper chambers) contract at a tremendously high rate and in an irregular pattern, the patient has atrial fibrillation. When the two lower chambers beat in an irregular pattern, the patient has ventricular fibrillation [3].

An electrocardiogram (ECG) is defined as an electrical signal which represents the heart's cardiac activity. The most important waves responsible for the creation of a usual ECG are normally the P, QRS and T waves. The P, QRS and T waves are related to the atrium's depolarization, ventricular depolarization and ventricular polarization, respectively. In general, the signal which relates to the atrium polarization, is merged with the QRS one. What is observed here is that the ECG beat's form can dynamically change. It is also highly correlated with the type of pathology. The R-wave that manifests the depolarization process of the ventricle is the highest amplitude of a single cycle of a normal ECG $[4,5]$. RR is the time intervals between successive R-waves, and the series of RR intervals is known as RR tachogram. Thus, in this time series, variability has been largely used as a heart function's measure which is known as heart rate variability (HRV). By investigating HRV signal, patients who are subject to cardiovascular accidents or death could be identified.

HRV signal is a non-stationary signal which describes the variations between consecutive heartbeats, and its changes can be interpreted as a current or upcoming disease. Furthermore, the interaction between sympathetic and parasympathetic activities in autonomic nervous system which controls cardiovascular system is evaluated using HRV.

Different mathematical methods exist to analyze HRV. The most common one is the Fourier transform which is limited to stationary signals. Wavelet transform analysis is one of the choices which can help to quantify HRV in non-stationary conditions. Wavelet transform (WT) represents a mathematical method used to study non-stationary signals [6]. The wave- let transform is comparable with the Fourier transform with a different merit function. The main difference is that Fourier transform decomposes the signal into cosines and sines but wavelet transform uses functions which are localized in both the Fourier and real spaces. This property makes wavelet transform an appropriate method for processing medical signals [7].

In the category of evolutionary algorithms (EA), which generate solutions to optimize problems using methods inspired by natural evolution including inheritance, mutation, selection and crossover, Genetic algorithm (GA) stand out the point [8]. It is one of the best methods to solve the problems which are little known. GA is a general algorithm and it works well in any search space. In artificial intelligence field, a GA is a search heuristic that mimics the process of natural selection. This heuristic is regularly used to generate useful solutions to search problems and optimization.

Support vector machines (SVM) are a group of supervised learning methods which can be applied to classification or regression. SVM denotes an extension to nonlinear models of the generalized portrait algorithm developed [9].

The purpose of this study is the separation of normal and abnormal ECG signals from each other; abnormal ECGs include ventricular tachycardia and arterial fibrillation. In this paper, a real-time Pan-Tompkins algorithm is used for QRS complex detection which reliably recognizes QRS complex based upon digital analyses of slope, amplitude and the width. Wavelet transform is then applied to the detected RR intervals to study the HRV.

Recently, some innovations of computer-aided algorithms have been successfully applied in automatic arrhythmia classification. Martis et al. [10] proposed SVM, neural network $(\mathrm{NN})$ and probabilistic neural network (PNN) classifiers for automated diagnosis of 5 types of arrhythmias including: fusion betas, nonectopic beats, ventricular ectopic beats, supra- 
ventricular ectopic beats and unclassifiable and paced beats. Discrete wavelet transform (DWT) sub-bands after independent applying three-dimension reduction algorithms including; principal component analysis (PCA), independent component analysis (ICA) and linear discriminant analysis (LDA) $[2,11,12]$ were fed to classifiers. It has yielded an accuracy of 99.28\%. Balasundaram et al. [13] presented a two-level binary classifier to at first extract VT and categorize the overlap zone between OVF and DVF using wavelet analysis of surface ECGs. The proposed method classifies OVF and DVF with an accuracy of $80 \%$. Prasad et al. [14] have used non-linear features of higher order spectra (HOS) for the detection of atrial flutter, atrial fibrillation and normal sinus rhythm beats. ICA employed for data reduction and the ICA coefficients are used as input of K-nearest neighbor (KNN), classification and regression tree (CART) and NN classifiers to evaluate the best automated classifier and reported $97.65 \%$ of accuracy. Sumathi et al. [15] proposed a neuro-fuzzy inference system (ANFIS) for the classification of 5 most important types of ECG signals containing: normal sinus rhythm (NSR), ventricular fibrillation (VF), atrial fibrillation (AF), pre-ventricular contraction (PVC) and ventricular flutter (VFLU) myocardial ischemia. The classification accuracy of $98.24 \%$ is obtained.

In the current study, we present a methodology to categorize the input ECG beat into 3 classes containing Normal sinus rhythm, atrial fibrillation and ventricular arrhythmia. The wavelet transform is used to extract features from RR intervals and then genetic algorithm is subjected to select the best features. These selected features are used for the classification of ECGs using SVM network.

\section{Material and Methods}

The proposed method includes preprocessing, feature extraction, feature dimension reduction by GA and the classification of arrhythmia using SVM. Figure 1 shows a block

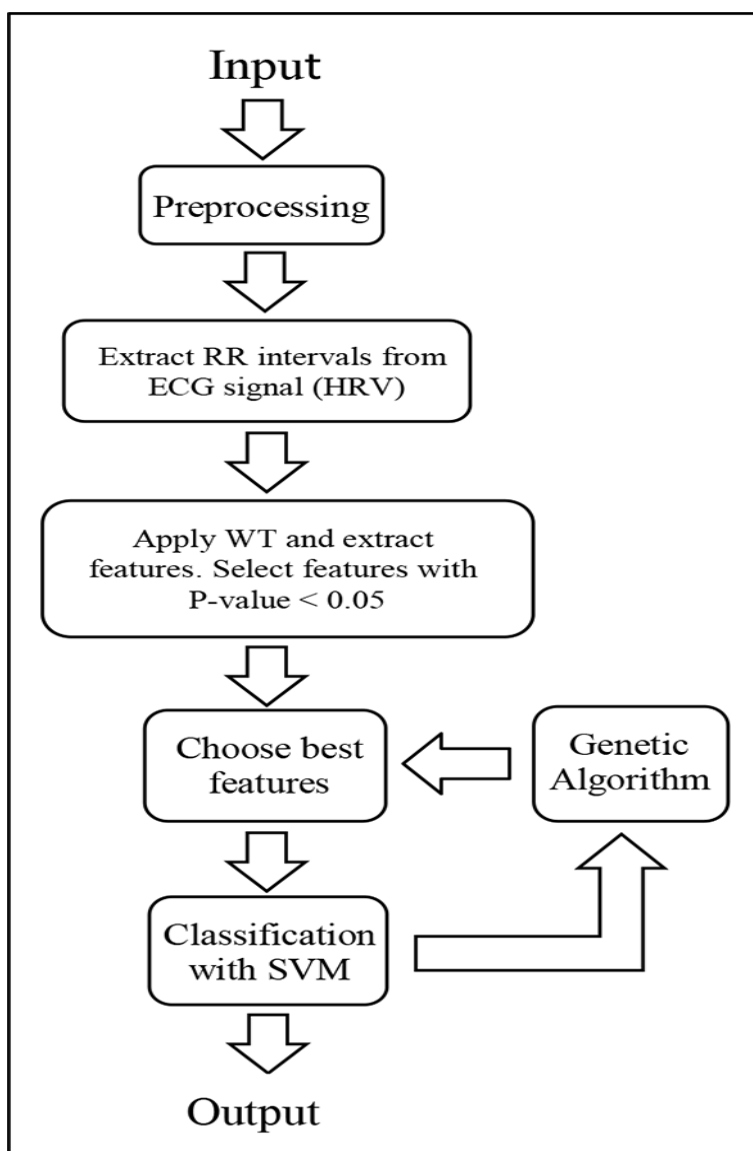

Figure 1: Block diagram of proposed classification algorithm.

diagram of the proposed algorithm. In this project, we used MIT-BIH normal sinus rhythm database, MIT-BIH arrhythmia database, MIT-BIH atrial fibrillation database and MIT$\mathrm{BIH}$ malignant ventricular arrhythmia database. The MIT-BIH arrhythmia database contains 48 excerpts of two-channel ambulatory ECG recordings, each record is approximately 30 minutes long with sampling frequency of $360 \mathrm{~Hz}$, MIT-BIH Normal sinus rhythm database includes 18 long-term ECG recordings, MIT-BIH atrial fibrillation database includes 25 long-term ECG recordings with sampling frequency of $250 \mathrm{HZ}$ and MIT-BIH malignant ventricular arrhythmia database contains 22 thirty-five minute records that sampled at $250 \mathrm{~Hz}$. In total, $53 \mathrm{ECG}$ records contain 18 normal rhythms (Figure 2), 20 subjects with 
atrial fibrillation (Figure 3 ) and 15 cases with ventricular tachycardia (Figure 4) which have been used [16].

\section{Preprocessing}

Filters play a key role in ECG acquisition to remove selected frequencies from an incoming signal and to minimize artifacts. The frequency band of the ECG signal is 0.05 to $100 \mathrm{~Hz}$. ECG Noise sources include muscle noise due to electrode motion during measurement, power-line interference $(60 \mathrm{~Hz})$, baseline wander due to respiration and $\mathrm{T}$ waves with high-frequency characteristics similar to QRS complexes. Power line noise can affect the $\mathrm{P}$ and $Q$ waves of ECG signal, generating errors during the diagnosis of myocardial infarction or arrhythmia. During the measurement of
QRS complex interval or QT interval, $60 \mathrm{~Hz}$ noise can cause errors by distorting the ECG which are vital diagnostic parameters [17]. For power-line noise removal, a least means square (LMS) adaptive filter is deployed by setting the notch filter of the $60 \mathrm{~Hz}$ band or the $60 \mathrm{~Hz}$-component as a reference signal. Here, the $60 \mathrm{~Hz}$-component is included $[6,16,18]$. In the next step, baseline wander which results from respiration (the low-frequency noise below $1 \mathrm{~Hz}$ ) and has the same frequency band as the ST segment of the ECG signal is precisely removed. Due to the fact that muscle artifacts are wildly distributed in the frequency band, they can generate distortions in the ECG signal when noises are removed. Figures 5 and 6 show the noisy and filtered ECG signals in time and frequency domain, respectively.

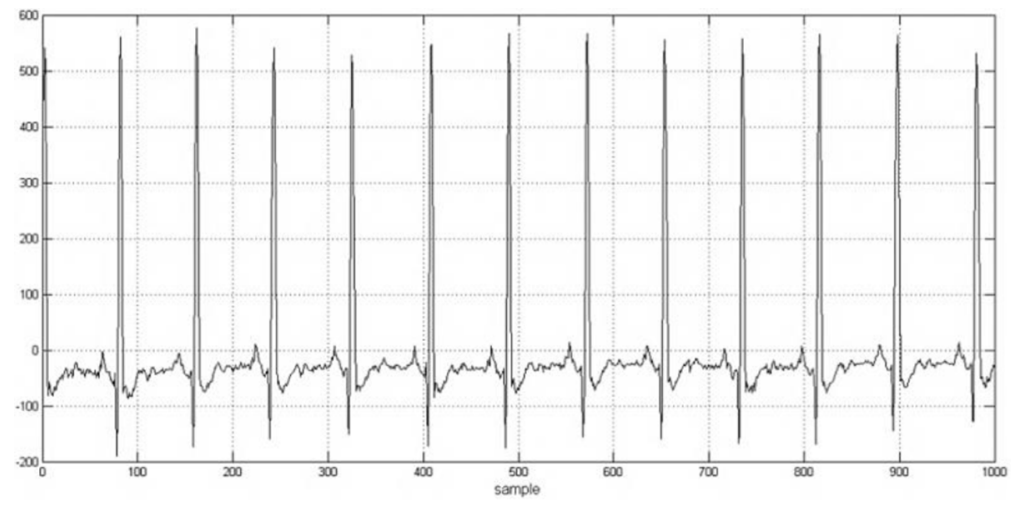

Figure 2: ECG of Normal Patient.

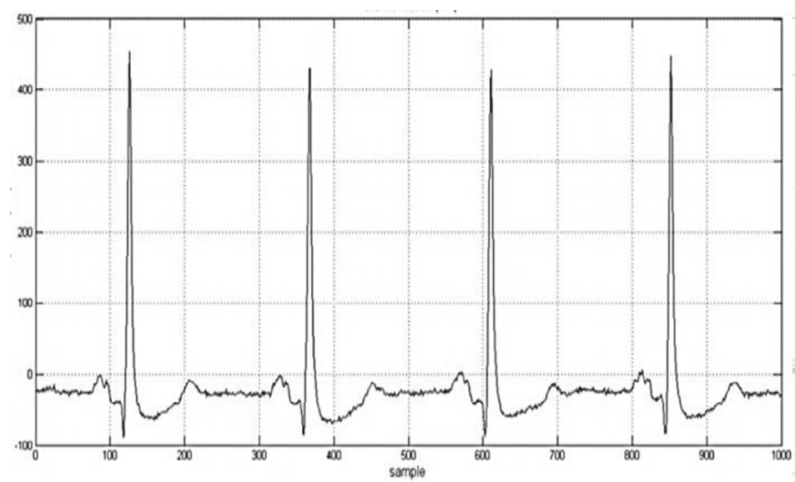

Figure 3: ECG of Patient with Arterial Fibrillation.

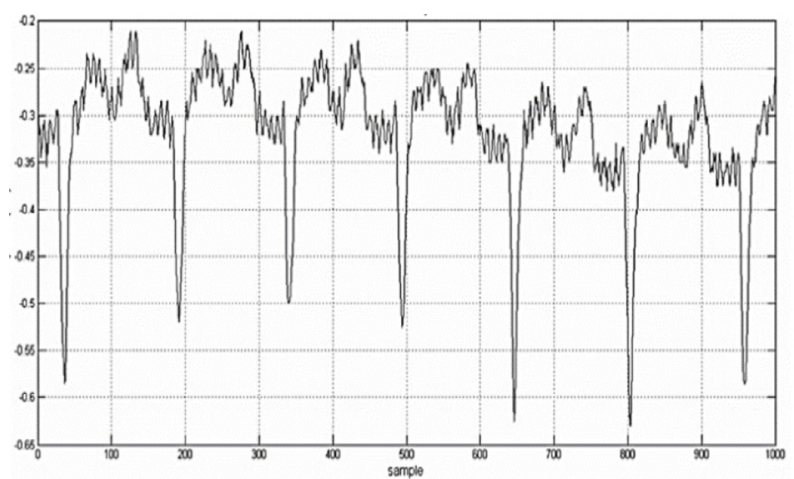

Figure 4: ECG of Patient with Ventricular Tachycardia. 

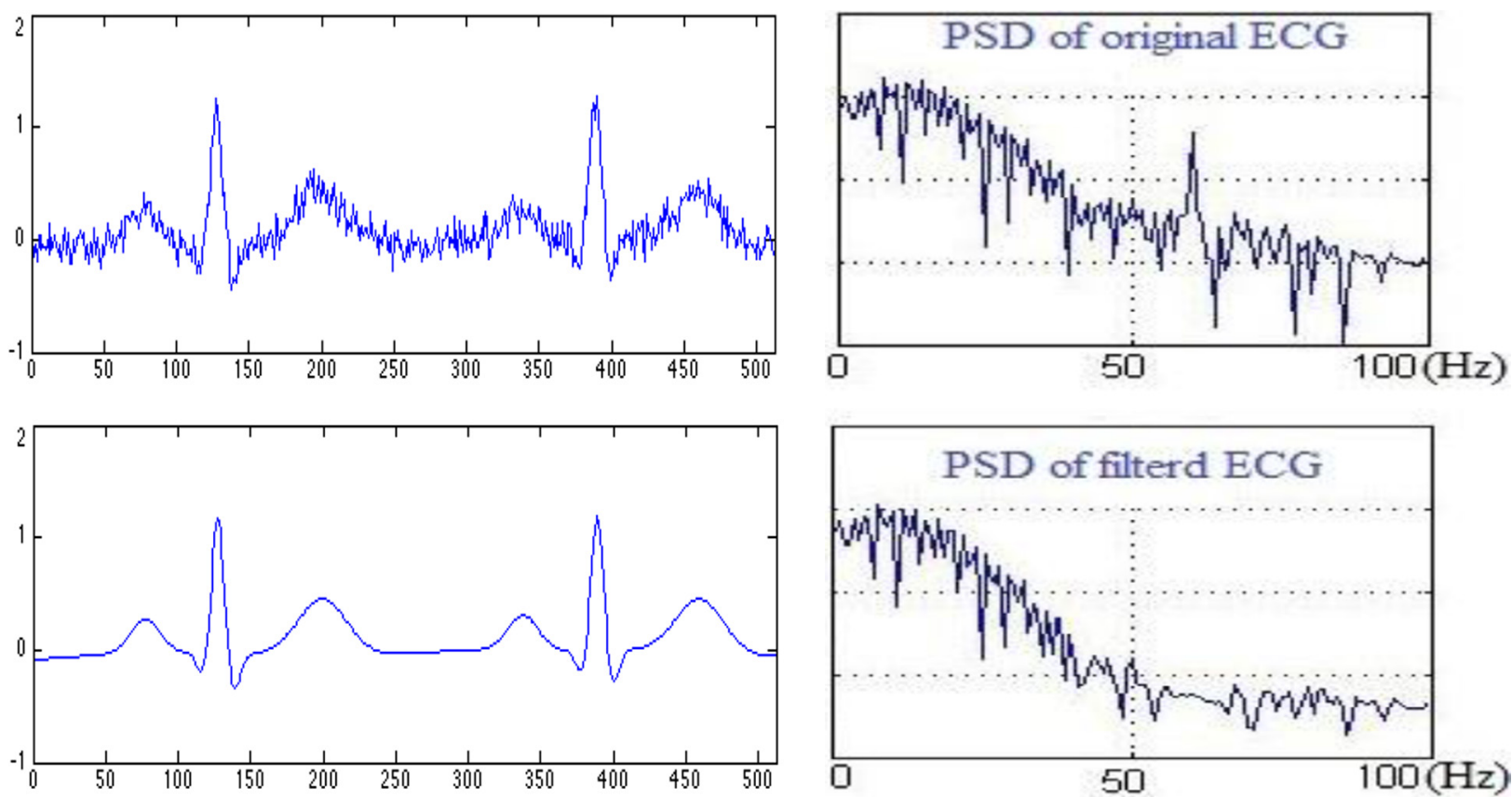

Figure 5: Noisy and Filtered ECG.

Figure 6: Power spectral density.

\section{QRS Detection}

Physiological variability of QRS complexes makes the QRS extraction difficult. Automatic detection of QRS complex is necessary for the efficient detection of beat-to-beat intervals (RR) from ECG. Accuracy of RR sequence is vital for reliable analysis of heart rate variability (HRV) that is widely considered to provide a simple quantitative and noninvasive assessment of cardiac-autonomic function in health and disease states. We used an on-line real-time QRS detection algorithm which is known as Pan-Tompkins algorithm [18]. This algorithm reliably detects QRS complexes using slope, amplitude and width information. In this algorithm, the ECG is differentiated to obtain the QRS slope information using the following formula:

$y[n]=\frac{1}{8}(2 x[n]+x[n-1]-x[n-3]-2 x[n-4])$

for making the data points positive and highlighting the higher frequencies signal is squared:

$$
y[n]=x^{2}[n]
$$

the algorithm performs sliding window integration to extract waveform feature information,

$\mathrm{y}[\mathrm{n}]=\frac{1}{\mathrm{~N}}(\mathrm{x}[\mathrm{n}-(\mathrm{N}-1)]+\mathrm{x}[\mathrm{n}-(\mathrm{N}-2)]+\ldots+\mathrm{x}[\mathrm{n}])$

where $\mathrm{N}$ is the size of the sliding window which depends on the sampling rate. The rising edge of the integrated waveform corresponds to the temporal location of the QRS.

In the last step, two thresholds are adjusted. The higher one identifies the peak of the signal. The lower one is used when no peak has been distinguished by the higher threshold at a certain time interval. So the algorithm has to search back in time for a missing peak. When a new peak is identified, then this peak is classified as a signal maximum if it beats the higher threshold or as a noise peak otherwise. In order to extract a QRS complex, the integrated waveform and the filtered signal are investigated and different values for the above thresholds are used. To be marked as a correct 
QRS complex, a peak must be recognized as a QRS in both integrated and filtered waveforms. After peak detection, heart rate variability (HRV) is analyzed to provide an indication of cardiovascular health.

\section{HRV Analysis}

HRV can be analyzed in frequency or time domain. Time domain parameters of HRV are the easiest as they are based on common statistical methods. Simple time domain variables include the mean normal-to-normal interval (all intervals between adjacent QRS complexes), the average heart rate, the difference between the shortest and the longest NN interval, the difference between night and day heart rates, to name but a few. The standard deviation (the square root of variance) of the $\mathrm{NN}$ interval (SDNN) is the most common extracted variable. SDNN reflects all cyclic components which are responsible for variability in the period of recording due to the fact that variance is mathematically equivalent to total power of spectral analysis. Other commonly used statistical variables calculated from segments of the whole monitoring era include the standard deviation of the average $\mathrm{NN}$ interval calculated over short periods (SDANN), usually $5 \mathrm{~min}$, which is an approximation of the changes in heart rate due to cycles longer than $5 \mathrm{~min}$. The most commonly used measures extracted from interval differences include the square root of the mean squared differences of successive NN intervals (RMSSD) [19].

Fast Fourier Transformation (FFT) is commonly used to extract and analyze dynamic changes in signals in general. The fundamental principle of this method is based on the fact that every signal can be described by the summation of a set of harmonic waves which results in the complete waveform. FFT can be used to decompose a waveform into its sine and cosine constituents [17, 20,21].

Since the first and second statistical moments of HRV signal are invariant in a temporal window of a few minutes, it could be served as a stationary signal. Therefore, Fourier transform or autoregressive batch analyses are adequate spectral decomposition techniques. In other situations, where transitory changes in the signal could happen, these approaches are not the most suitable choices for the analysis, because the signal acquires non-stationary characteristics.

To overcome this inconveniency, there are several methods including short time Fourier transform (STFT), discrete wavelet analysis, time-frequency distributions and time-varying analysis [22]. Transforming HRV signal from time domain to wavelet representation in timefrequency domain allows achieving good recognition results.

In this article, Haar wavelet transform with order 4, is used to extract the features of HRV time series. Discrete wavelet transform (DWT) gives a decomposition of a given signal into a set of approximate (A $i)$ and detailed (Di) coefficients of level $i(i=1, \ldots, n)$

The DWT of a signal $\mathrm{x}$ is calculated by passing it through a series of filters (Figure 7). First the samples are passed through a lowpass filter with impulse response $g$. The input signal and the filter response function should be convolved to form the output:

$$
y[n]=x[n] * g[n]=\sum_{k=-\infty}^{\infty} x[k] \cdot g[n-k]
$$

A high-pass filter $\mathrm{h}$ is also used to decompose the signal, simultaneously. The outputs give the detail coefficients (from the high-pass filter) and approximation coefficients (from the low-pass filter). These high-pass and lowpass filters are related to each other, and they are known as a quadrature mirror filter (Figure 7).

$$
\begin{aligned}
& y_{\text {low }}[n]=\sum_{k=-\infty}^{\infty} x[k] \cdot h[2 n-k] \\
& y_{\text {high }}[n]=\sum_{k=-\infty}^{\infty} x[k] \cdot g[2 n-k]
\end{aligned}
$$

Since only half of each filter output characterizes the signal, this decomposition has halved the temporal resolution. However, each 


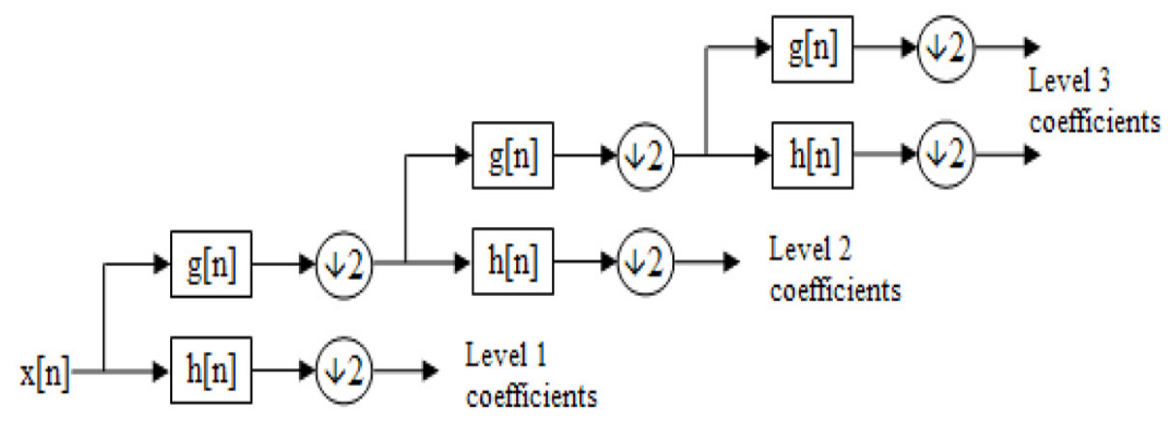

Figure 7: A level 3 filter bank.

output has half the frequency band of the input so the frequency resolution has been doubled. With the subsampling operator $\downarrow$ :

$$
(y \downarrow k)[n]=y[k n]
$$

The above summation can be written more concisely:

$$
\begin{aligned}
& y_{\text {low }}=(x * g) \downarrow 2 \\
& y_{\text {high }}=(x * h) \downarrow 2
\end{aligned}
$$

However, computing a complete convolution $x * g$ with subsequent downsampling

would excess computation time.

In this study, each HRV signal is decomposed into four levels using the wavelet transform. For each level, several features including Shannon's entropy, mean, variance, kurtosis and spectral component $\beta$ were extracted from $\operatorname{Di}(i=1, \ldots, 4)$ and A4 [23, 24]. In total, it is possible to obtain 23 wavelet-based features from each HRV time series [25].

The spectral component $\beta$ is defined as the slope that gets the spectral density over several decades of frequency. In particular, the exponent $\beta$ is 0 for white noise and 2 for Brownian motion. Recently, the discrete wavelet transform method based on orthonormal wavelet decomposition has been introduced to estimate the exponent $\beta$ and is calculated from the log-scale plot of the variance versus the resolution $[25,26]$.

$\beta_{W v 2^{j}-W v 2^{j^{-1}}}=\log _{2}\left(\operatorname{variance}\left(W v_{2^{j}}\right)\right)-\log _{2}\left(\operatorname{variance}\left(W v_{2^{j-1}}\right)\right)$
Where $W v$ is wavelet coefficient of level $2^{j}$.

Selection of Best Features by Genetic Algorithm

In this study, GA is used for feature selection which is an effective algorithm in solving large-scale problems, and can be used to find an optimal feature subset. In GA, individuals are typically represented by n-bit binary vectors. Each of these individuals would represent a feature subset in a feature selection problem. It is supposed that the quality of each candidate solution can be assessed using a fitness function. In this study, the accuracy of classification is considered as a fitness function. GA parameters are adjusted as follows:

- Population size: 500

- Number of generation: 250

- Probability of mutation: 0.05

- Probability of crossover: 0.7

- Crossover strategy: Random single point

- The mutated bits of selected chromosomes: 0.1

\section{Classification}

In this study, normal and abnormal HRV signals and automatically distinguished from each other using SVM. SVM introduced by Vapnik and Cortes in 1995, are considered as a powerful classifier. Like other classifiers, the aim of SVM is to find out a decision surface that splits the dataset into two sets. All data lying on each side of the decision surface will 
be classified as members of one class. SVM is able to find the unique decision surface which also has a maximum distance or margin between the two datasets. In other words, SVM is able to find the optimal decision surface [27]. We need to select features which provide the best performance result as input features to SVM.

Student's t test was used to analyze the parameters associated with arrhythmia and normal HRV, and $\mathrm{P}$ value of less than 0.05 was considered significant, so the features are ranked in the increasing order of their estimated p-values [23]. To have a good choice on features applying Genetic algorithm (GA), features with $\mathrm{p}$ value more than 0.05 are removed. Therefore, we have 13 out of 23 features for GA input.

\section{Evaluation}

An objective method is needed to evaluate the performance of the proposed classification algorithm. The performance of the classification capability of each parameter set in discriminating between normal and two types of arrhythmias was evaluated by several objective indices. For the evaluation of our proposed algorithm, we used accuracy, sensitivity, specificity and precision defined as follows:

sensitivity $=\frac{T P}{(T P+F N)} \quad$ specificity $=\frac{T N}{(T N+F P)}$

precision $=\frac{T P}{(T P+F P)}$ accuracy $=\frac{T P+T N}{(T P+F P+F N+T N)}$

where, TP, TN, FP and FN denotes true positive, true negative, false positive and false negative respectively.

\section{Results}

Our experiments were conducted using a database of 53 ECG records including18 normal rhythms, 20 subjects with atrial fibrillation and 15 cases with ventricular tachycardia. P-values of student's t-test for wavelet based SVM were calculated. Table1 shows p-value of each feature. By using this approach, 13 significant
Table 1: P-Value $(p \leqq 0.05$ was considered statistically significant).

\begin{tabular}{cl} 
Feature & P-Value \\
\hline Average Approximation & $0.00028^{* *}$ \\
\hline Variance Approximation & 0.11744 \\
\hline Entropy Approximation & 0.08234 \\
\hline Kurtosis Approximation & $0.00118^{* *}$ \\
\hline Average Detail1 & 0.17907 \\
\hline Variance Detail1 & 0.12206 \\
\hline Entropy Detail1 & 0.06278 \\
\hline Kurtosis Detail1 & $0.00001^{* *}$ \\
\hline Average Detail2 & $0.00013^{* *}$ \\
\hline Variance Detail2 & 0.11259 \\
\hline Entropy Detail2 & $0.04140^{* *}$ \\
\hline Kurtosis Detail2 & $0.00021^{* *}$ \\
\hline Average Detail3 & $0.00108^{* *}$ \\
\hline Variance Detail3 & 0.10115 \\
\hline Entropy Detail3 & 0.06240 \\
\hline Kurtosis Detail3 & $0.00047^{* *}$ \\
\hline Average Detail4 & $0.00366^{* *}$ \\
\hline Variance Detail4 & $0.02307^{* *}$ \\
\hline Entropy Detail4 & $0.03679^{* *}$ \\
\hline Kurtosis Detail4 & $0.00024^{* *}$ \\
\hline B1_component & $0.00878^{* *}$ \\
\hline B2_component & 0.10044 \\
\hline B3_component & 0.39829 \\
\hline$* *$ P-Value $\leqq 0.05$ & \\
\hline
\end{tabular}

features (all with $\mathrm{p}<0.05$ ) among the total of 23 features were selected. However, we need to reduce the features dimension in order to have more significant results. For this purpose, GA has been utilized to select 4 most important ones from these 13 features. The most important features are: kurtosis detail1, entropy detail2, average detail3 and B1_component, which demonstrate significant differences in these three classes.

Figure 8 illustrates box and whisker plots of these different features. Evidently, kurtosis detaill in ventricular tachycardia has lower values $(4.3 \pm 1)$ in comparison with two normal $(8.84 \pm 3.34)$ and arterial fibrillations $(8.76 \pm 2.34)$, entropy detail2 in arterial fibril- 


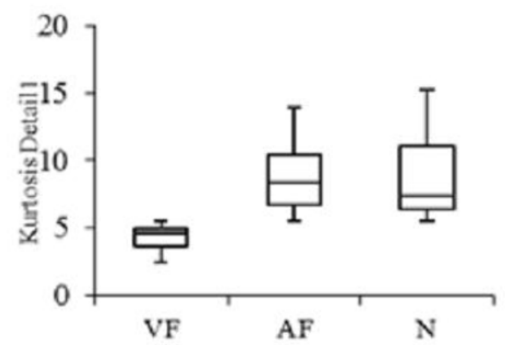

(A)
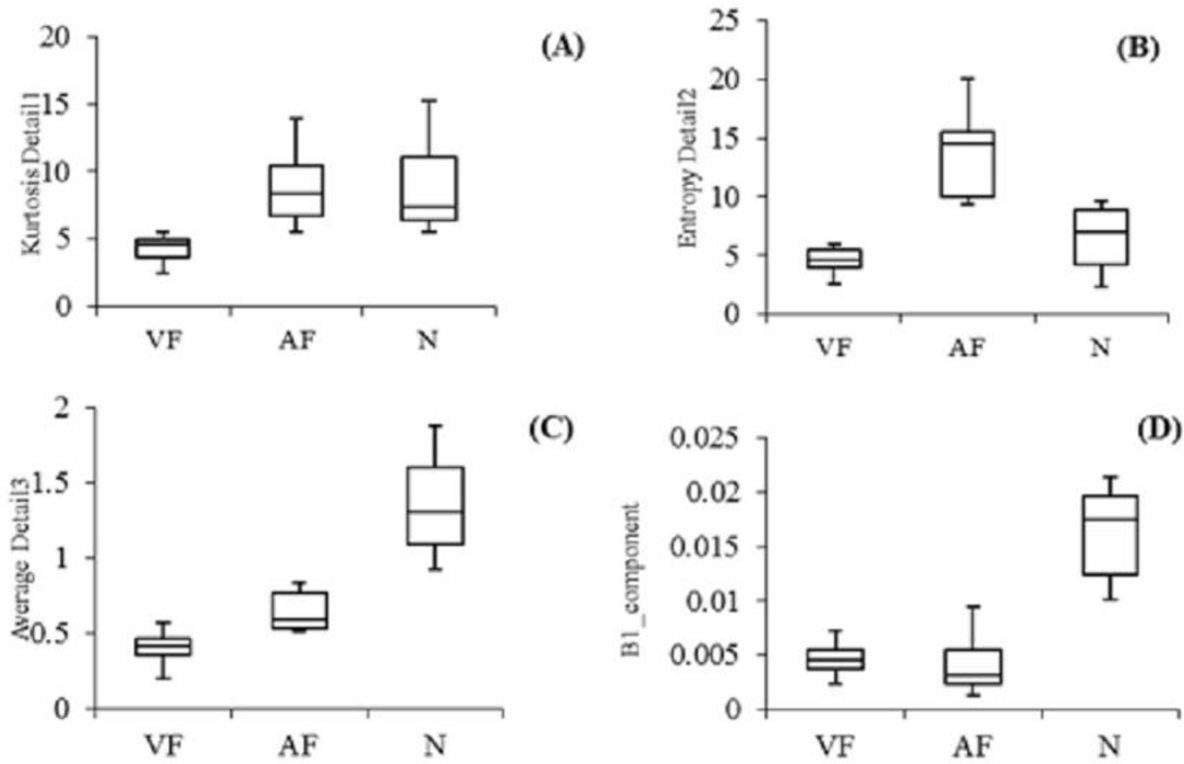

(C)

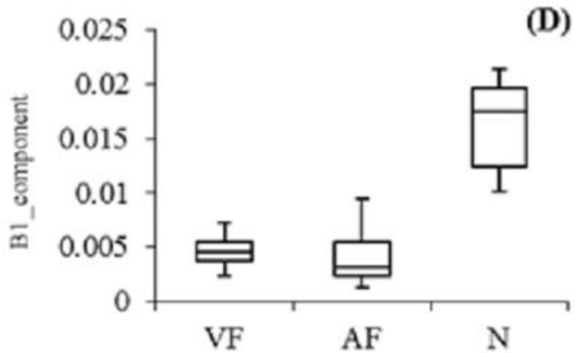

Figure 8: Box-plots and whisker diagrams of different wavelet based features for normal (N), Arterial fibrillation (AF) and Ventricular fibrillation (VF). The box represents the values from lower to upper quartile and the central line is representative of the median. The whiskers are expanded from lower to upper values. (A) Kurtosis Detail1, (B) Entropy Detail2, (C) Average Detail3 and (D) B1_component.

lation has higher values $(13.62 \pm 3.32)$ in comparison with normal $(6.56 \pm 2.6)$ and ventricular tachycardia (4.52 \pm 1.01$)$, average detail3 and B1_component are features that in normal ECG have higher values versus two other types of arrhythmia; average detail3 (N: $1.36 \pm 0.31$ vs VT: $0.38 \pm 0.14$ and AF: $0.64 \pm 0.12)$ and B1_component $(\mathrm{N}$ : $0.016 \pm 0.004$ versus VT: $0.0046 \pm 0.0014$ and AF: $0.004 \pm 0.0023$ )

The combined features with $p$-value $<0.001$ and the most important features selected by GA were considered for discrimination between two types of cardiac arrhythmia and normal rhythm. We assigned $70 \%$ of the data as the training group and the remaining 30\% was used to train the SVM classifier. A nonlinear SVM with a polynomial kernel was exploited as the classifier. The performance of the classification capability of parameters set in discriminating between normal and between two types of cardiac arrhythmia with and without feature reduction was evaluated by several objective indices; the results are summarized in Table 2.

Using the combination of all four features with p-value $<0.001$, the accuracy of SVM for classifying was $95.54 \%$; the sensitivity, $96.31 \%$; the specificity, $93.01 \%$ and the precision value, $92.40 \%$. After removing less important features by GA, a combination of kur-

Table 2: Results by dimension reduction using GA and without dimension reduction.

\begin{tabular}{ccccc} 
& accuracy & sensitivity & specificity & precision \\
\hline Without dimension reduction & $95.54 \%$ & $96.31 \%$ & $93.01 \%$ & $92.40 \%$ \\
\hline Dimension reduction using GA & $97.14 \%$ & $97.54 \%$ & $96.9 \%$ & $97.64 \%$
\end{tabular}


tosis detail1, entropy detail2, average detail3 and B1_component, evaluation of the SVM for classifying arrhythmias from normal beats results in accuracy of $97.14 \%$; sensitivity of $98.40 \%$; specificity of $96.98 \%$ and precision $97.94 \%$.

\section{Discussion}

In this paper, we presented a classification scheme that consists of the GA feature reduction method and the SVM using wavelet based features extracted from HRV for classifying two types of cardiac arrhythmia and normal rhythm.

Various features have been proposed in the literature for the classification of ECG arrhythmia. The classification performance of any ECG arrhythmia classification system strongly depends on feature extraction, feature reduction and the classification algorithm. $\mathrm{Hu}$ et al. [28] have proposed a patient-adaptable cardiac arrhythmia classification method. They have combined self-organizing maps (SOM), learning vector quantization (LVQ) along with the mixture-of-experts (MOE) method. Experiments were conducted with 48 records from MIT/BIH ECG arrhythmia database and achieved an accuracy of $94.0 \%$ in the classification of ventricular ectopic beats (VEB's). In MOE method, an expert cardiologist annotated a specific segment of patient ECG to mix two classifiers and realize patientadaptation. Inan et al. [29] have combined morphological wavelet transform and time domain information of 18 records of the MIT/ $\mathrm{BIH}$ arrhythmia database to detect premature ventricular contractions (PVCs) and obtained $98.3 \%$ and $97.4 \%$ of accuracy. Übeyli et al. [30] proposed multilayer perceptron neural network (MLPNN) to classify four types of ECG beats containing normal beat, congestive heart failure beat, ventricular tachyarrhythmia beat and atrial fibrillation beat. Lyapunov exponents, wavelet coefficients and the power levels of power spectral density (PSD) values obtained by eigenvector methods of the ECG signals were used for discriminating four types of ECG beats. The method applied on 20 beats of ECG signal of MIT-BIH database that is a small dataset to classify ECG beats into four different classes. Ince et al. [31] employed fully connected artificial neural networks designed for each patient for the detection of ventricular ectopic beats (VEBs) and supra-VEBs (SVEBs). They used 48 records of MIT/BIH arrhythmia database to classify seven beats and accuracy of $96.06 \%$ was reported. Martis et al. [10] used Support Vector Machine (SVM), neural network (NN) and probabilistic neural network (PNN) classifiers for the detection of five arrhythmia classes as recommended by AAMI on the MIT-BIH arrhythmia database. Three-dimension reduction methods were applied to DWT sub bands and an accuracy of $99.28 \%$ has yielded. Balasundaram et al. [13] presented singular value decomposition (SVD) using wavelet analysis of surface ECGs to classify ventricular arrhythmias. The proposed method classifies OVF and DVF with an accuracy of $80 \%$. Sumathi et al. [15] proposed a Neuro-Fuzzy Inference System (ANFIS) for the classification of five most important types of ECG signals and the classification accuracy of $98.24 \%$ is obtained.

This paper presented an effective combination of wavelet-based parameters extracted from HRV. In this study, we have experimentally used GA to reduce the dimensionality of DWT coefficients. In this method, the input ECG beat was categorized into 3 classes containing Normal sinus rhythm, atrial fibrillation and ventricular arrhythmia.

Our study reveals that an effective combination of wavelet-based parameters in an SVM classification scheme could improve the diagnostic performance of two types of arrhythmia with acceptable accuracy of $97.14 \%$.

The results demonstrate that removing less important features by GA results in increased accuracy, sensitivity, specificity and precision. This improvement can be caused by better performance of SVM classification with reduced 
number of features.

\section{Conclusion}

ECG signal depicts the electrical activity of the heart providing vital information on the cardiac health. In this study, we proposed wavelet-SVD based method to classify arrhythmias using genetic algorithm. In the present work, from selection of best features by genetic algorithm, two classes of cardiac arrhythmias are detected with good classification accuracy and class specific accuracy.

A comparative analysis with the related existing methods illustrates that the proposed method has higher potential in the classification of AF and VF. The attempt to classify ECG signals has been successfully achieved. The proposed method has shown a promising sensitivity of $97.54 \%$ which indicates that this technique is an excellent model for computer aided diagnosis of cardiac arrhythmias.

The advantage of the proposed classifier using GA is not only its simplicity but also its simple implementation. Furthermore, the proposed methodology can be used in arrhythmia monitoring systems, cardiac pacemakers and telemedicine applications.

Presently, there is a rising burden of cardiovascular diseases around the world; hence, accurate detection of cardiac health is essential to improve the quality of life. The developed automated tool can help medical community in healthcare diagnosis with considerable reliability and precision.

\section{Acknowledgment}

The authors would like to acknowledge the Department of Biomedical Engineering and Medical Physics, Faculty of Medicine, Shahid Beheshti University of Medical Sciences.

\section{Conflict of Interest}

\section{None}

\section{References}

1. Tavassoli M, Ebadzadeh MM, Malek H. Classification of cardiac arrhythmia with respect to ECG and
HRV signal by genetic programming. Canadian Journal on Artificial Intelligence, Machine Learning and Pattern Recognition. 2012;3:1-8.

2. Mansoory MS, Ashtiyani M, Tajik H, editors. Cardiac motion evaluation for disease diagnosis using ICA basis neural network. Computer Science and Information Technology-Spring Conference, 2009. IACSITSC'09. International Association of; 2009: IEEE.

3. Behbahani S, Asadi S, Ashtiyani M, Maghooli K, editors. Analysing optical flow based methods. Signal Processing and Information Technology, 2007 IEEE International Symposium on; 2007: IEEE.

4. Dubin D. Rapid Interpretation of EKG's: USA: Cover Publishing Company, 1996; 1996.

5. El Khansa L, Nait-Ali A. Parametrical modelling of a premature ventricular contraction ECG beat: comparison with the normal case. Comput Biol Med. 2007;37:1-7. doi.org/10.1016/j.compbiomed.2005.07.006. PubMed PMID: 16310174.

6. Kheder G, Kachouri A, Taleb R, Ben Messaoud M, Samet M. Feature extraction by wavelet transforms to analyze the heart rate variability during two meditation techniques. Advances in Numerical Methods: Springer; 2009. p. 379-87.

7. Ashtiyani M, Behbahani S, Asadi S, Birgani PM, editors. Transmitting encrypted data by wavelet transform and neural network. Signal Processing and Information Technology, 2007 IEEE International Symposium on; 2007: IEEE.

8. Vali M. Sub-Dividing Genetic Method for Optimization Problems. arXiv preprint arXiv:1307.5679. 2013.

9. Cortes C, Vapnik V. Support-vector networks. Machine learning. 1995;20:273-97. doi.org/10.1007/ BF00994018.

10. Martis RJ, Acharya UR, Min LC. ECG beat classification using PCA, LDA, ICA and discrete wavelet transform. Biomedical Signal Processing and Control. 2013;8:437-48. doi.org/10.1016/j. bspc.2013.01.005.

11. Ashtiyani M, Asadi S, Birgani P, Khordechi E, editors. EEG Classification using Neural networks and Independent component analysis. 4th Kuala Lumpur International Conference on Biomedical Engineering 2008; 2008: Springer.

12. Ashtiyani M, Asadi S, Birgani PM, editors. ICAbased EEG classification using fuzzy C-mean algorithm. Information and Communication Technologies: From Theory to Applications, 2008. ICTTA 2008. 3rd International Conference on; 2008: IEEE.

13. Balasundaram K, Masse S, Nair K, Umapathy K. 
A classification scheme for ventricular arrhythmias using wavelets analysis. Med Biol Eng Comput. 2013;51:153-64. doi.org/10.1007/s11517-0120980-y. PubMed PMID: 23132525.

14. Prasad H, Martis RJ, Acharya UR, Min LC, Suri JS. Application of higher order spectra for accurate delineation of atrial arrhythmia. Conf Proc IEEE Eng Med Biol Soc. 2013;2013:57-60. doi.org/10.1109/ embc.2013.6609436. PubMed PMID: 24109623.

15. Sumathi S, Beaulah HL, Vanithamani R. A wavelet transform based feature extraction and classification of cardiac disorder. J Med Syst. 2014;38:98. doi.org/10.1007/s10916-014-0098-x. PubMed PMID: 25023652.

16. Moody GB, Mark RG. The impact of the MIT-BIH arrhythmia database. IEEE Eng Med Biol Mag. 2001;20:45-50. PubMed PMID: 11446209

17. von Borell E, Langbein J, Despres G, Hansen S, Leterrier $\mathrm{C}$, Marchant-Forde $\mathrm{J}$, et al. Heart rate variability as a measure of autonomic regulation of cardiac activity for assessing stress and welfare in farm animals -- a review. Physiol Behav. 2007;92:293-316. doi.org/10.1016/j.physbeh.2007.01.007. PubMed PMID: 17320122.

18. Pan J, Tompkins WJ. A real-time QRS detection algorithm. IEEE Trans Biomed Eng. 1985;32:2306. doi.org/10.1109/TBME.1985.325532. PubMed PMID: 3997178.

19. Gritti I, Defendi S, Mauri C, Banfi G, Duca P, Roi GS. Heart rate variability, standard of measurement, physiological interpretation and clinical use in mountain marathon runners during sleep and after acclimatization at $3480 \mathrm{~m}$. Journal of Behavioral and Brain Science. 2013;3:26. doi.org/10.4236/ jbbs.2013.31004.

20. Ashtiani M, Asadi S, Goudarzi PH, editors. A New Method in Transmitting Encrypted Data by FCM Algorithm. Information and Communication Technologies, 2006. ICTTA'06. 2nd; 2006: IEEE.

21. Birgani PM, Ashtiyani M, Asadi S, editors. MRI segmentation using fuzzy c-means clustering algorithm basis neural network. Information and Communication Technologies: From Theory to Applications, 2008. ICTTA 2008. 3rd International Conference on; 2008: IEEE.

22. Ranganathan G, Bindhu V, Rangarajan DR. Signal processing of heart rate variability using wavelet transform for mental stress measurement. Journal of Theoretical \& Applied Information Technology. 2010;11.

23. Radivojac P, Obradovic Z, Dunker AK, Vucetic S, editors. Feature selection filters based on the permutation test. European conference on machine learning; 2004: Springer.

24. Bajpai P, Kumar M. Genetic algorithm-an approach to solve global optimization problems. Indian Journal of computer science and engineering. 2010;1:199-206.

25. Wornell GW, Oppenheim AV. Estimation of fractal signals from noisy measurements using wavelets. IEEE Transactions on signal processing. 1992;40:611-23. doi.org/10.1109/78.120804.

26. Khandoker AH, Begg RK, Palaniswami M, editors. Estimating Falls Risk in the Elderly: A Wavelet Based Multiscale Analysis. Electrical and Computer Engineering, 2006. ICECE'06. International Conference on; 2006: IEEE.

27. Li G, Chung WY. Detection of driver drowsiness using wavelet analysis of heart rate variability and a support vector machine classifier. Sensors (Basel). 2013;13:16494-511. doi.org/10.3390/ s131216494. PubMed PMID: 24316564. PubMed PMCID: 3892817.

28. Hu YH, Palreddy S, Tompkins WJ. A patient-adaptable ECG beat classifier using a mixture of experts approach. IEEE Trans Biomed Eng. 1997;44:891900. doi.org/10.1109/10.623058. PubMed PMID: 9282481.

29. Inan OT, Giovangrandi L, Kovacs GT. Robust neural-network-based classification of premature ventricular contractions using wavelet transform and timing interval features. IEEE Trans Biomed Eng. 2006;53:2507-15. doi.org/10.1109/ TBME.2006.880879. PubMed PMID: 17153208.

30. Übeyli ED. Statistics over features of ECG signals. Expert Systems with Applications. 2009;36:875867. doi.org/10.1016/j.eswa.2008.11.015.

31. Ince T, Kiranyaz S, Gabbouj M. A generic and robust system for automated patient-specific classification of ECG signals. IEEE Trans Biomed Eng. 2009;56:1415-26. doi.org/10.1109/ TBME.2009.2013934. PubMed PMID: 19203885. 